\title{
Rules for authors
}

\section{GENERAL PROVISIONS}

Journal "Acta Naturae» publishes experimental research papers and reviews, and also discussion papers, mini-reviews, short communications, which are related to most topical problems of the fundamenta and practical life sciences and biotechnologies.

Journal is published by Park Media Publishing Company in Russian and English languages.

Edition board of the journal "Acta Naturae" would like to ask authors to follow the following rules. Manuscripts, which are not consistent with those rules, will be sent back to authors without consideration.

The maximal size of review together with tables and references should not be more than 60000 characte (it is about 40 pages of A4 format and with 1.5 lines interval, Times New Roman, size 12), and 16 figures.

The size of experimental research paper should not exceed 30000 characters, (20 pages of A4 format, including tables and references). The number of figure should not exceed 10. Papers of larger size could be accepted only in case of preliminary agreement with the editors.

Short communication should include task design experimental data and conclusions. The size of shor communication should not exceed 12000 characters (8 pages of A4 format, including tables and references, no more than 12 references). Number of figures should not exceed 4 .

Manuscript should be presented as a set of files: main test, summary, list of references, comments to figures, tables must be in Microsoft Word 2003 for Windows format, figures must be in JPG or TIF format each figure should represented as a separate file.

Manuscript should be sent to editor board as an electronic version; it is possible to do it on a CDROM.

\section{MANUSCRIPT DESIGN}

Manuscript should have:

Title of the manuscript. It must not be very long or very short or low-informative. It should represent the main result, the essence and the novelty of the work.

Authors initials and surnames with the references to the organisations in which authors work. Author, which is responsible for the communication with the editorial board and correction, should be indicated by the reference, and his correspondent e-mail should be represented on the bottom of the first page.

Full name of the scientific organisation and agency.

Summary. Structure of summary must be very clea and reflect following: task design; experimental methods, possibility of the practical application, new possible tasks and problems. 20 lines.

Keywords. Keywords should reflect: the subject of research, method, object, specificity of this work.

Abbreviations.

Introduction.

Chapter "Experimental part."

Chapter "Results and discussion."

Chapter "Conclusions." At the end of this chapter names of organisations, that provided financial support, should be indicated (and in brackets number of grants should be indicated).

Chapter "References." References in text should be given in square brackets, for example [1].

RECOMMENDATION ABOUT TEXT FORMATTING:

We recommend using Microsoft Word 2003 for Windows editor.

Font should be Times New Roman, and standart size is 12 .

Interval between lines is 1.5

We do not recommend using more that one space between words.

Strictly do not use automatic reference option, automatic hyphenation or automatic block of hyphenation automatic list, automatic indention etc.

It is recommended to use Word for making tables (Table - Add Table) or MS Excel. Tables, which are created manually (by using a lot of empty spaces, but not cells), should not be used.
Always, it must be an empty space between surnames and initials: A.A. Ivanov (except from the names of authors in the title of the manuscript, in this situation empty spaces must be between initials as well, i.e A. A. Ivanov)

Everywhere in the text, except the date of manuscript's arrival, all dates should be in format "date. month.year," according the examples: 02.05.1991, 26.12 .1874 and so on.

Do not use full stop after title of the manuscript, authors names, addresses, headings and subheading, titles of tables, comments to figures, dimensionalities ( $\mathrm{s}$ for second, $\mathrm{g}$ for gram, min for minute, $\mathrm{h}$ for hours, d for days, ${ }^{\circ}$ for degrees).

Full stop must be after: footnotes (and particularly in tables), comments to the table, short annotations, abbreviations ( $y$. for years, t. melt. for the temperature of melting, but not for inferior indexes: $T_{\text {melt }}$ for temperature of melting, $T$ for temperature of phase transition). Exception: mln for million does not need full stop.

For decimal fractions use full stops, but not commas (0.25, but not 0.25$)$

Dash "-" must be between two empty spaces, symbols for "minus", "interval" or "chemical bound" do not need to be between two empty spaces.

Use only " $x$ " as a symbol for multiplication. Use symbol " $x$ " only in case if there is a number in the right from his symbol. Symbol "." is used for the complex compounds in chemical formulas, and also for the noncovalent complexes (DNA-RNA and so on)

Do not use inverted commas for quotes.

All numerical data should be represented as tables. In formulas, use the letters of Latin and Greek alphabets.

Latin names of genera and species must be in italic font, for higher taxons use regular font. For the names of viruses and bacteriophages in Latin transcription use regular font.

Names of genes (except the yeast genes) should be in italic font, for names of proteins use regular font

Names of nucleotides ( $, T, G, C, U$ ), amino acid residues (Arg, lle, Val etc.) and phosphates (ATP, AMP etc) should be written in Latin transcription using regular font.

Numeration of nucleotides and amino acid residues should be written without hyphen (T34, Ala89).

Use international system of units $\mathrm{SI}$ for measurement

For molecular mass, use daltons (da, kda, mda).

For the number of nucleotide pair, use abbreviations

(b.p, t.b.p.).

Biochemical terms (and particularly, names of enzymes) should be according the international rules of IUPAC.

Using of abbreviations of terms in the text should be minimized.

Repetition of the same data in text, tables and graphs is not allowed.

\section{RULES FOR ILLUSTRATIONS}

Figures for manuscripts should be represented as separate files.

The resolution of illustrations must be $300 \mathrm{dpi}$ or higher for the colourful or halftone pictures, and 600 dpi for the black-white illustrations.

It is not allowed to keep in files the ways of clipping paths that are not in use, and also to keep additional channels (Path and Alpha Channel), and also to use additional layers.

File's formats should be in Adobe Photoshop up to version 7.0. (including this version), TIFF, JPG.

\section{REVIEWING, PREPARATION TO PUBLISHING}

\section{AND THE QUEUE FOR PUBLICATION}

Papers will be published accordingly to the process of receiving. The queue for the publications will be established accordingly to the date of approval for publication. Members of Editorial Board have a right to recommend the papers, which were classified as a high priority and had high marks of reviewers, to the faster publication. Manuscripts, which arrived to Edi- torial Board, are reviewed by the experts from Editorial Board, and, if it is required, could be sent to the external review.

The prerogative of Editorial Board is to choose the reviewer. The manuscript will be sent to the review of the experts in this area of research, and the Editorial Board will make a decision about the fate of this manuscript: it could be accepted as it is, it could need an improvement and it could be rejected.

If manuscript does not fit to the above requirements, it could be rejected by the decision of Editorial Board.

If manuscript was sent back to authors for improvement as a decision of reviewers and editors, it will be reviewed second time, and after that Editorial Board will consider the possibility of the publication of this manuscript again. The date of the arrival of manuscript and the date of the acceptance of this manuscript to publishing will be indicated at the beginning of the paper.

Returning of the manuscript for improvement back to the authors does not mean that this manuscript is accepted to publishing. After improvement, this manuscript will be considered by Editorial Board again. The author should return back the text after improvement together with the original variant of manuscript and also with the answers to all of the comments. The date of arrival of the final version of the manuscript to Editorial Board is considered as date of arrival of the manuscript.

The improved manuscript should arrive back to Editorial Board during 1 week after author accepted comments; otherwise this manuscript will be considered as a newly arrived.

Editorial Board uses e-mail on the all of the steps of the communication with authors; authors must be very careful with the e-mail that is indicated in the manuscript, and in case of any changes immediately provide the information about changes to the Editorial Board.

After the approval of the layout of the journal, Editorial Board will send the proofreading to authors via e-mail in the PDF file.

On the step of correction, it is not possible to change the text, figures of tables. If it is necessary, this question will be considered by Editorial Board.

\section{REFERENCES FORMAT}

For books: author's surname and initials, full name of the book, the place og publishing, publishing company, year of publication, volume or issue and total number of pages.

For periodical issues: author's surname and initials, the name of the journal, year of publication, volume, number, firs and last page of the paper.

Bressanelli S., Tomei L., Roussel A., et al/ / Proc. Natl. Acad. Sci. USA. 1999. V. 96. P, 13034-13039 (if there are more than 5 authors), and all authors, if there are less than 5 authors.

References to the author's abstracts of the dissertations should contain author's surname and initials, title of the dissertation, the place were work was done, year of the dissertation's defence.

References to patents should contain surnames and initials of authors, the type of the patent's document (certificate of recognition or patent), number, the name of the country in which document was issued, index of international classification of inventions, year of the patent's issue.

The list of references should be represented on the separate page.

Tables should be represented on the separate page.

Comments to figures should be represented on the separate page.

For the communication with Editorial Board use e-mail addresses: rpetrov@strf.ru, knorrevd@gmail.com,vsychev@strf.ru, tel.: (495) 727-38-60, (495) 930-80-05 